\title{
SCRATCHING AND BRITTLE FRACTURE OF SEMICONDUCTOR IN-SITU SCANNING ELECTRON MICROSCOPE
}

\author{
K. Wasmer ${ }^{1}$, Pouvreau, $C^{1-2}$, Ballif, $\mathrm{C}^{3}$ and Michler, $\mathrm{J}^{1}$ \\ ${ }^{1}$ EMPA, Material Science Technology \\ Feuerwerkerstrasse 39, 3602 Thun, Switzerland \\ ${ }^{2}$ Swiss Federal Institute of Technology Lausanne (EPFL), Station 9, 1015 Lausanne, Switzerland \\ ${ }^{3}$ University of Neuchâtel, Institute of Microtechnique (IMT), A.-L. Breguet 2, 2000 Neuchâtel, \\ Switzerland \\ kilian.wasmer@empa.ch
}

\begin{abstract}
The miniaturisation and the complexity of optical laser devices has been the focal centre of many industries in recent years. One key issue of the processing is the dicing of laser bars and devices. This process is constituted by the two crucial operations which are (1) the scratching and (2) the cleavage. The goal of the first operation is to create a subsurface crack aligned with a cleavage plane. This crack is required to be able to control the crack initiation and propagation, even in brittle materials such as Gallium-Arsenide (GaAs) so that atomically flat surfaces can be achieved [1]. Unfortunately, scribing semiconductors with a diamond tip does not only induce the defect needed but also some undesirable features such as radial cracks (chevrons) and particles. The purpose of the second operation is the separation of the laser bars and/or individual lasers. In order to investigate these two operations, two special apparatus, working inside a Scanning Electron Microscope (SEM), were employed. It was found that the scratching velocity and tip direction (edge first or face first) have only little influence on the onset of radial cracks and chips. However, the tip direction affects the size and number of the chips. Additionally, the depth of the initial crack, a, for subsequent cleavage, can be related to the applied load, $P_{S}$, by the following power law: $a^{3 / 2} \propto P_{S}$. $P_{S}$ has been found to be inversely related to $P_{C}$. Finally, the scatter measured in $P_{C}$ is dependent on the scratching load $P_{S}$. This is due to the fact that at high $P_{S}$ values, the median cracks are not constant throughout the scratch length and some deeper points exist which act as stress concentrations.
\end{abstract}

\section{Introduction}

In the high power lasers diodes industries, the separation of the laser diodes bars and/or the individual lasers has become both a quality issue and an economic necessity. In order to perform this operation a number of in-house procedures have been developed. The most common procedure is the scratching and cleavage of the different devices [2-3]. However, although this procedure adopts some basic principles, often the fundamental knowledge to understand the effects of the different operations is missing. In recent years, scientists have either been investigating the scratching operation, (especially on polymer and/or glasses [4-6]), or the cleavage operation in semiconductors [7-11]. In this work a correlation between both phenomena is made for Gallium-Arsenide (100) wafer, designated as GaAs.

This paper was divided in two main parts since scratching and cleavage are studied. The first one concentrates on the scratching of GaAs with two types of three sided diamond tips. This includes the investigation of (a) the occurrence of Lateral Crack (LC) and particle generation based on either in-situ SEM and conventional experiments and (b) the influence of the load on the initial defect, designed as Median Crack (MC). The second part focuses on the cleavage operation. In this section, the correlation between the scratching load, $P_{S}$, and the critical load at which the $\mathrm{MC}$ is initiated, $P_{C}$, is analysed. This relationship is also examined in term of the stress intensity factor $K_{l}$ since the crack initiation will strongly depend on this fracture mechanisms parameter. Two simple models were used which are taken from the literature as a basis [12-15]. 


\section{Experiments and Materials}

Instruments

Two types of nano-scratching instruments were employed in this work. The first one is a Nanoindenter-XP (MTS/Nanoinstruments, Oak Ridge, TN) equipped with a lateral force measurement option. This instrument was used to induce the initial defect by scratching at loads ranging from 5 up to $200 \mathrm{mN}$. The second is a home-made apparatus responding to the specification necessary to work inside a SEM. All technical information regarding its construction and specifications are given in [16] and so only a brief description is given here. Figure 1 shows a picture of the SEM nanoindenter. The piezoactuator has a nominal range of $20 \mu \mathrm{m}$ and a maximal force of $400 \mathrm{~N}$. The sample positioning table includes a $X, Y$ and $\theta$ tables. The $X$ table is controlled by a stick-slip actuator and can be used for scratch length up to $10 \mathrm{~mm}$. In contrast, the $Y$ table has a range of only $20 \mu \mathrm{m}$ since this axis is driven by a stack piezoactuator. The $\theta$ table is also controlled by a stick-slip and allow to orientate the specimen with precision.

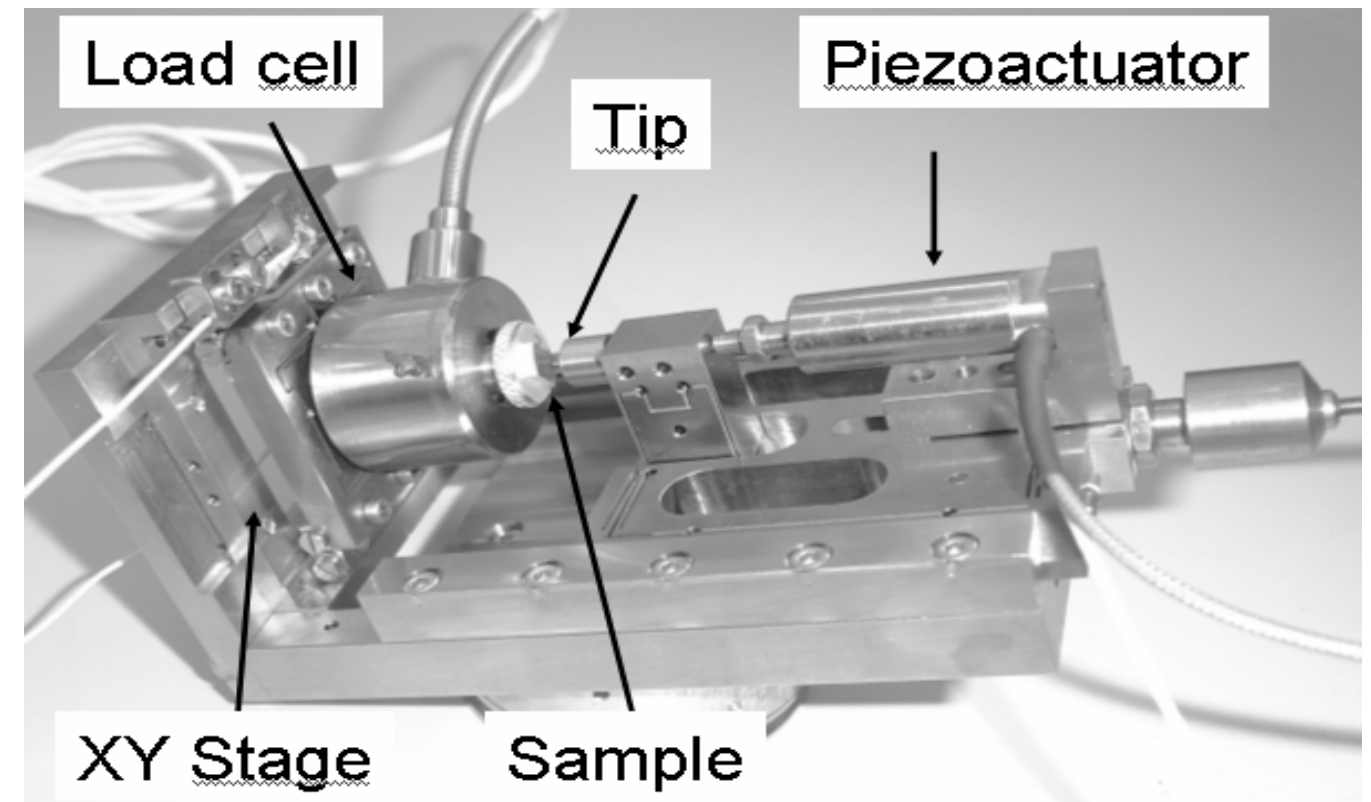

Figure 1: Picture of the nano-scrachting device. The $X-Y$ table controls are respectively stick-slip (10 $\mathrm{mm}$ stroke) and piezo controlled ( $20 \mu \mathrm{m}$ range). The $\mathrm{z}$ axis is controlled by a piezoactuator and has a range of $20 \mu \mathrm{m}$.

The cleavage experiments were carried out with an apparatus integrated into a SEM chamber. It has a stroke of several hundred of micrometers and was "displacement-controlled". Its displacement control was better than $100 \mathrm{~nm}$ and the force applied by the cleaving rod on the wafer was acquired simultaneously.

The investigation of the subsurface crack network was performed by looking at cross-sections which were prepared with a Focussed Ion Beam (FIB) FEI Strata DB 235.

\section{Materials}

In this present work, the material used was pure Gallium-Arsenide (GaAs) (100) wafers doped with $1-2 \cdot 10^{18}$ Silicon Atoms $/ \mathrm{cm}^{3}$. GaAs is part of the III-V semiconductor family and is an anisotropic material. Hence, its material properties will dependence on crystal orientation and the principal characteristics are given in Table 1. The preferential cleavage plane is known to be $\{110\}$ which is consistent with the lowest $K_{I C}$ value in Table 1 [1, 7-11]. The average fracture strength of GaAs wafers measured from micromechanical cantilever beam structure tests is more or less $2.4 \mathrm{GPa}$ [17]. Hence, large forces are required to cleave such wafer if no initial defect is induced. All GaAs (100) wafers employed had a miscut of $2^{\circ}$ to the [110] direction, as typically used for epitaxial growth. 


\begin{tabular}{|c|c|c|c|c|c|c|}
\hline $\begin{array}{c}\text { Young's Modulus } \\
{[\mathrm{MPa}]}\end{array}$ & \multicolumn{2}{c|}{$\begin{array}{c}\text { Poisson } \\
\text { coefficient }\end{array}$} & \multicolumn{2}{c|}{$\begin{array}{c}\boldsymbol{K}_{/ \mathrm{C}} \\
{\left[\mathrm{MPa} \cdot \mathbf{m}^{1 / 2}\right]}\end{array}$} & Reference \\
\hline$\langle 100\rangle$ & 89.3 & $\langle 100\rangle$ & 0.31 & $\{100\}$ & 0.43 & {$[11]$} \\
\hline$\langle 110\rangle$ & 125.0 & & & $\{110\}$ & 0.31 & {$[11]$} \\
\hline$\langle 111\rangle$ & 144.0 & & & $\{111\}$ & 0.45 & {$[11]$} \\
\hline & & & & $\{110\}$ & $0.44-0.46$ & {$[8]$} \\
\hline
\end{tabular}

Table 1: GaAs material properties

\section{Diamond tips}

Two types of diamond tips were utilised for scratching the GaAs. They were a Cube Corner (CC) and a Berkovich. The equivalent conical angle of the CC tip is 84.6 degrees and has the same shape as the corner of a cube. The tip radius was measured to be around $0.5 \mu \mathrm{m}$. The Berkovich tip, also a three sides, has a total angle of $142.5^{\circ}$ and an apex angle of $65.3^{\circ}$. The tip radius was measured to be roughly $2.5 \mu \mathrm{m}$ by Atomic Force Microscopy (AFM). The surface cracks and particle generation were studied with the CC tip either in-situ or with the Nanoindenter-XP. The scratches were made with the Berkovich tip to observe the subsurface cracks. The reason is that this tip does not guarantee a decent view of the scratching zone in the SEM. Finally, the analyses of crack initiation and propagation were performed only with the Berkovich tip.

\section{Experiments}

Two distinct scratching velocities were chosen to investigate its influence and they were taken to be 10 and $100 \mu \mathrm{m} / \mathrm{sec}$. For the correlation between $P_{S}$ and $a$, the scratch length, velocity and crystallographic direction were $3 \mathrm{~mm}, 100 \mu \mathrm{m} / \mathrm{s}$ and [110], respectively. In order to obtain sufficient measurements for a statistical study, five scratches were measured at five different locations and at eight different loads. The loads were 5, 15, 30, 50, 75, 100, 150 and $200 \mathrm{mN}$. Prior to the cleavage operation, scratches were produced with a length of $400 \mu \mathrm{m}$ to be closer to the industrial process. Then, the scratched wafers were clamped with vice (a toll having tow parts which can be moved together by tightening a screw so that an object can be held firmly). The distance between the scratch and the vice was more or less $200 \mu \mathrm{m}$. The displacement rate for all tests was 187.5 $\mu \mathrm{m} / \mathrm{s}$. Two to three cleavage tests were completed.

\section{Experimental Results of Scratching}

\section{Introduction}

It has already been observed that, by and large, 5 different phenomena occur when scratching semi-conductors [1] and glasses [4]. Figure 2, taken from [1], illustrated these 5 regimes. The first regime is purely elastic. It is followed by the elastoplastic regime. In the third region, subsurface cracking takes place which depends strongly on the tip geometry [1]. This includes the formation of lateral and median cracks. Then, in addition to them, radial cracks (chevrons) are created. Finally, creation of chips is observed.

In this work, it will be shown from inspection of the videos and pictures taken in-situ SEM that all regions exist for the cube corner and Berkovich tips.

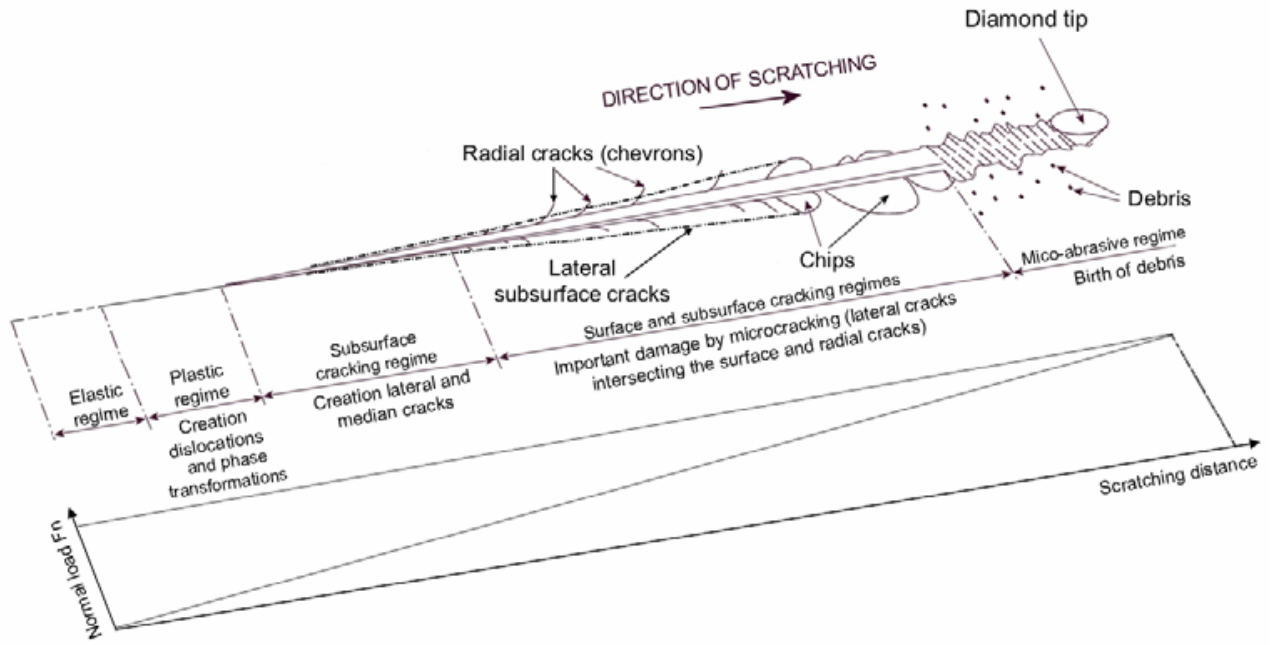

Figure 2: Typical scratch pattern of semiconductors generated by an increasing load along the scratch path (Taken from [1]) 


\section{Surface cracks}

The elastico-plastic region, the radial cracks and chips in Fig. 3 (a) - (c), respectively, can be related to Fig. 2. Besides, it is noticed that the radial cracks are created in most cases just behind the tip or sometimes on its side. These chevrons propagates in a straight line at the beginning as in Fig. 3 (b) and then grow in a curved shape ending more or less perpendicular to the scratch direction as illustrated in Fig. 3 (c). The origin of large particles is the intersection of the lateral and radial cracks and the detachment of chip as shown in Figs 3 (c) and 4 (b).

In this investigation, two scratching speed with the cube corner tip were chosen, which are 10 and $100 \mu \mathrm{m} / \mathrm{s}$, with respect to the most favourable cleavage planes. Additionally, scratches were performed with the edge of the diamond tip first as well as face first. It is found that the transitions between the regions 1 and 2 occurred at loads less than $50 \mu \mathrm{N}$ in all cases. In contrast, inspection of Table 2 indicates that the loads at which the radial cracks and chipping out occur are not very sensitive to the direction of the diamond tip and the scribing velocity. It is obvious from this table that, except one test, the first lateral cracks and first chips appear at loads ranging from 0.8 to $2.0 \mathrm{mN}$ and 4.0 to $8.0 \mathrm{mN}$, respectively. At loads where chipping out occur, it is observed through in-situ experiments that the number and size of chips are larger when scribing face first compared to edge first. Furthermore, it has been seen, when scratching edge first, that the chipping out takes only place in front of the tip whereas when scratching face first, it might take place behind the tip. The scratch does not follow a straight line when it is performed with the face first as shown in Fig. 4 (a). Finally, it was observed in some cases that it is possible to remove GaAs material from the surface wafer using the diamond tip in a similar manner as a cutting tool by generating chip of a length of several millimetres.

Scrutinizing the results acquired while scratching along [110], two major differences are visible. To start with, the directions of the radial cracks are always along [100] and [1,-00] directions as illustrated in Figs 3 (b), (c) and 4 (a). Secondly, no slip bands are visible on track sides at these magnifications, and this for both tip orientations. In conclusions, for GaAs, the type of diamond tip, the scratching direction and applied load influence slightly the patterns observed at the surface. Finally, although not shown here, depending on the tip geometry, the creation of chips is possible even in very brittle materials such as GaAs.

\begin{tabular}{|c|c|c|c|c|}
\hline $\begin{array}{c}\text { Crystallographic } \\
\text { direction of the wafer }\end{array}$ & Scratch direction of & $\begin{array}{c}\text { Scratching } \\
\text { tip } \\
{[\boldsymbol{\mu m} / \mathbf{s}]}\end{array}$ & $\begin{array}{c}\text { Critical loads } \\
\text { for radial cracks } \\
{[\mathbf{m N}]}\end{array}$ & $\begin{array}{c}\text { Critical loads } \\
\text { for first chips } \\
{[\mathbf{m N}]}\end{array}$ \\
\hline$[110]$ & Edge first & 10 & 7.0 & 12.5 \\
\hline$[110]$ & Edge first & 100 & 1.2 & 8.0 \\
\hline$[110]$ & Face first & 10 & 0.8 & 4.0 \\
\hline$[110]$ & Face first & 100 & 1.4 & 5.0 \\
\hline
\end{tabular}

Table 2: Results of critical load for first lateral cracks and first chips depending on scratching conditions for tests performed on the Nanoindenter-XP with the Berkovich diamond tip with a radius of $\approx 2.5 \mu \mathrm{m}$ along [110]
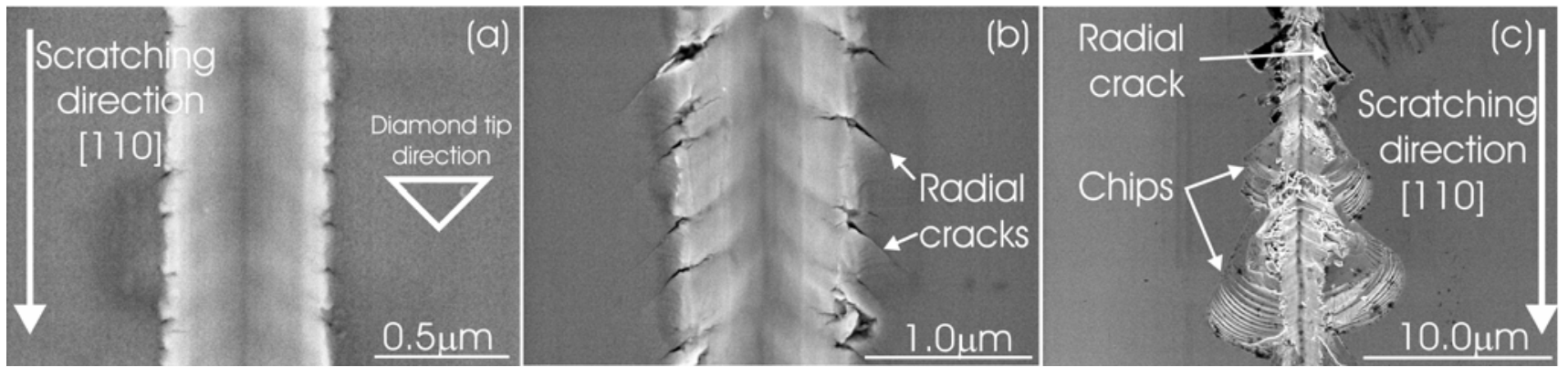

Figure 3: Characterisation of the different scratch patterns on GaAs made with a cube corner diamond tip with radius of $\approx 0.5 \mu \mathrm{m}$ and along [110] direction edge first. (a) Only plastic deformation is observed. (b) Radial crack appears and (c) Radial cracks and large chipping out are seen. 


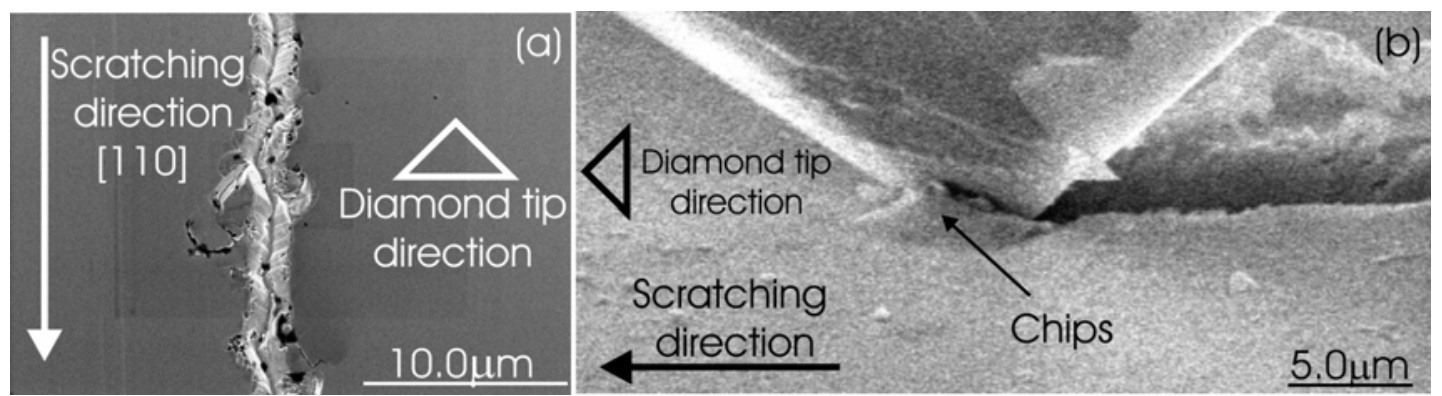

Figure 4: Characterisation of the different scratch patterns on GaAs made with a cube corner diamond tip with radius of $\approx 0.5 \mu \mathrm{m}$ and along [110] direction at $10 \mu \mathrm{m} / \mathrm{s}(\mathrm{a})$ face first and (b) edge first where radial crack and large particle are detached

\section{Correlation between the depth of the median crack and the scratching load}

As mentioned earlier, to cleave GaAs wafers containing no defect, large forces are necessary. The median crack is, most of the time, induced by scratching with all the consequences coming with such as surface cracks and chipping out. Recently, Wasmer et al. [1] showed that the scratching operation may, depending on the conditions, create a subsurface crack network. The investigation of this network was performed with a Focus lon Beam (FIB). Figure 5 shows an example of subsurface lateral (LC) and median cracks (MC). It is found that, as soon as the load reach a critical value, a $M C$ is generated along the preferred $\{110\}$ cleavage plane for GaAs. In this specific case, the MC has a depth of approximately $4 \mu \mathrm{m}$. In contrast, the shape of the LC is not reproducible and so have a more stochastic behaviour. It is interesting to notice from Fig. 5 that the MC does not start from the surface but at $\approx 0.6 \mu \mathrm{m}$ under the surface. This was explained by the fact that the initiation of the MC is located at the crossing of slip bands [1]. The micro-crack just below the scratch in Fig. 5 may be due to the plastic deformation induced by the shear stress inside the material. Similarly to the LC, these micro-cracks are randomly dispersed.

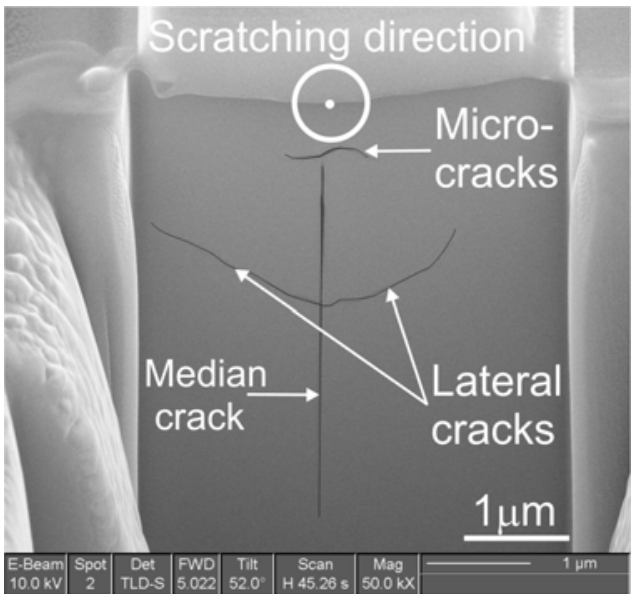

Figure 5: SEM cross-section prepared by FIB of a scratch made at a nominal force of $15 \mathrm{mN}$

In indentation, it is well known that there is a correlation between the normal applied force, $P$, and the depth of the median crack, $a$. This relation is derived from the relation between the stress intensity factor, $K_{l}$, and $a$. Assuming a MC with a semielliptical shape in pure tension, the relation between the residual stress intensity factor, $K_{R}$, and $a$ is given by Lawn [12]:

$$
K_{R}=\frac{\chi \cdot P}{a^{3 / 2}}
$$

where

$$
\begin{aligned}
& K_{R}=\text { residual stress intensity factor } \\
& P=\text { the normal applied load } \\
& \chi \quad=\text { dimensionless material constant } \\
& a=\text { depth of the median crack }
\end{aligned}
$$

Under certain condition, it is possible to obtain a stable equilibrium relation and so that Eq. (1) becomes:

$$
P \propto \chi \cdot a^{3 / 2}
$$

This behaviour has already been observed for little number of tests [18], but no real statistics has been performed. Although scratching is a much more complex phenomenon compared to indentation, the fracture mechanisms principle stands and thus, it is expected that Eq. (2) is still valid. The results of this investigation are shown in Fig. 6 and from this figure, two observations are seen. Firstly, it is observed that the minimum scratching load, $P_{S \text { Min, }}$ at which the initial defect is visible, is $15 \mathrm{mN}$. However it is known from the penetration curve that plastic deformation happens from $4 \mathrm{mN}$. Then, the model in Eq. (2) describes very well the relation between the scratching load and the size of the median crack. This is supported by the coefficient of determination $R^{2}=1$ characterising a "perfect" fit from a regression line based on least squares method made in Fig. 6 . 
While the results presented in Fig. 6 are limited between 15 and $75 \mathrm{mN}$, tests were carried out at loads ranging from 5 up to 200 $\mathrm{mN}$. An example of cross-section measurement is given in Fig. 7 . It is evident from Fig. 7 (a) that scratch at $30 \mathrm{mN}$ produce a nice smooth median crack with no or little damage on the surface. On the contrary, at $150 \mathrm{mN}$, the measures of the initial MC were not reliable and the surface was extremely damage by the diamond tip. As a result, they are locations where stress concentrations occur as can be seen in Fig. 7 (b). This was the case for loads comprised between 100 and $200 \mathrm{mN}$.

Finally, Figure 7 shows a good example of surface features that are seen depending on the cleavage condition. Since only atomically flat surfaces must be achieved, laser diodes bars and/or the individual lasers containing such features along the cleavage are rejected.

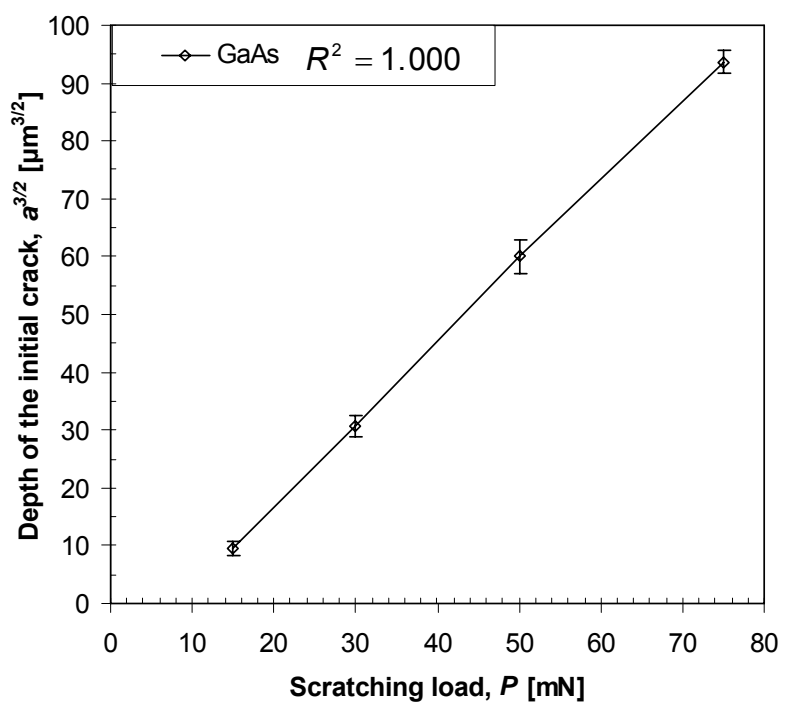

Figure 6: Depth of the median crack a vs. the normal applied load $P_{S}$ according to Eq. (2), $a^{3 / 2} \propto P_{S}$.

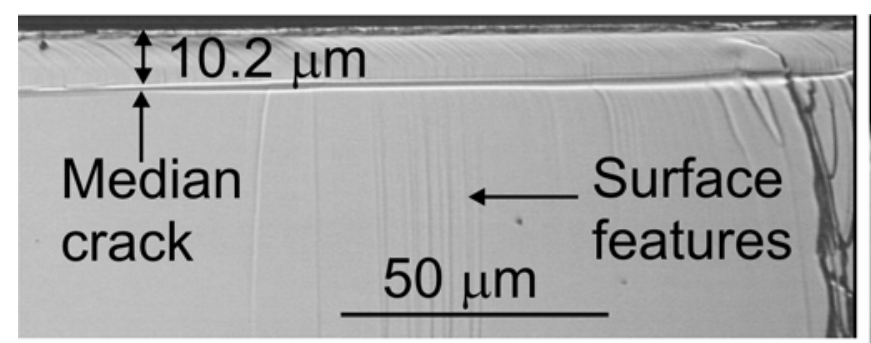

(a)

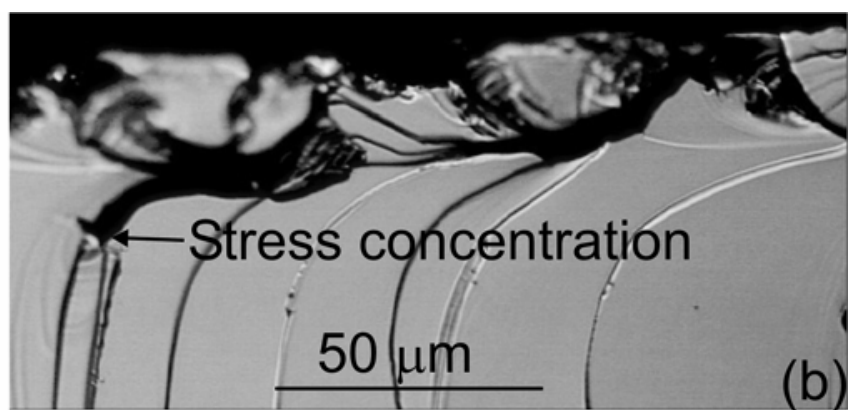

Figure 7: Typical view of the initial median crack created by scratching at (a) $30 \mathrm{mN}$ and (b) $150 \mathrm{mN}$ in GaAs. The scratches were performed with a Berkovitch diamond tip with a tip radius of $\approx 2.5 \mu \mathrm{m}$ scratching edge first at a speed of $100 \mu \mathrm{m} / \mathrm{s}$

\section{Experimental Results of Cleavage}

After the scratching operation was done to induce this initial defect (or median crack), the cleavage operation is investigated. This operation was carried out by applying a normal load through a stroke of hundred of micrometers as illustrated in Fig. 8, taken from [1]. The results of cleavage experiments are shown in Fig. 9. From this figure, it is seen that the load applied by the stroke on the wafer increases quite linearly until a critical load $P_{C}$ is reached. At $P_{C}$, the cleave initiates and the crack propagates at high speed. This is illustrated in the figure by the abrupt fall in the load-displacement curve. Then, depending on the condition, two cracking behaviours were perceived in Fig. 9. The first is that the crack does not propagate throughout the length of the wafer and so the additional crack growth occurs by steps as for $200 \mathrm{mN}$. The second is that the crack propagates throughout the length of the wafer at once as observed for the case of $5 \mathrm{mN}$.

Figure 9 presents the force-displacement curves recorded during cleaving of samples scratched at various loads. As expected from Fig. 6 , the critical load, $P_{C}$, decreases with an increase of the scratching load. Furthermore, the two cracking behaviour mentioned in the previous paragraph are observed. 


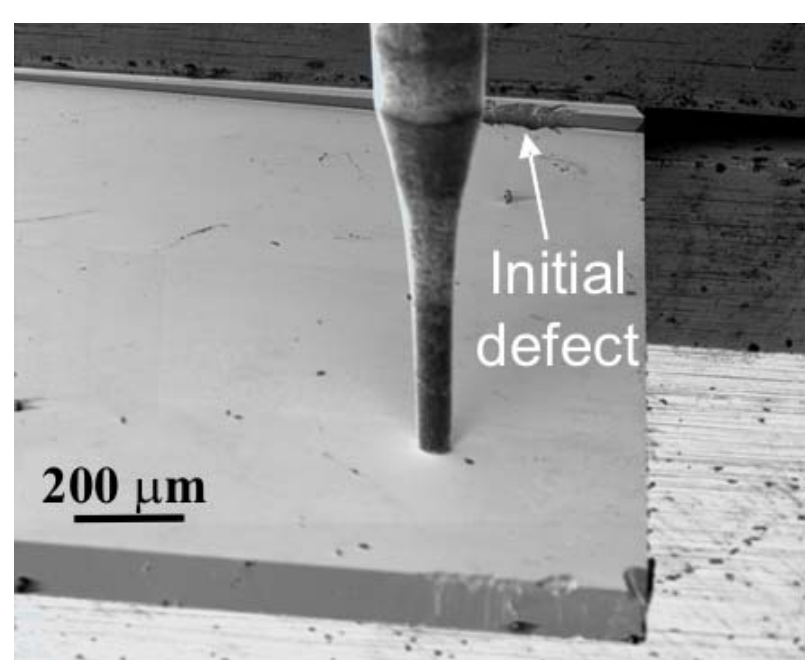

Figure 8: Picture of the "displacement-controlled" stroke employed for the in-situ cleavage operation

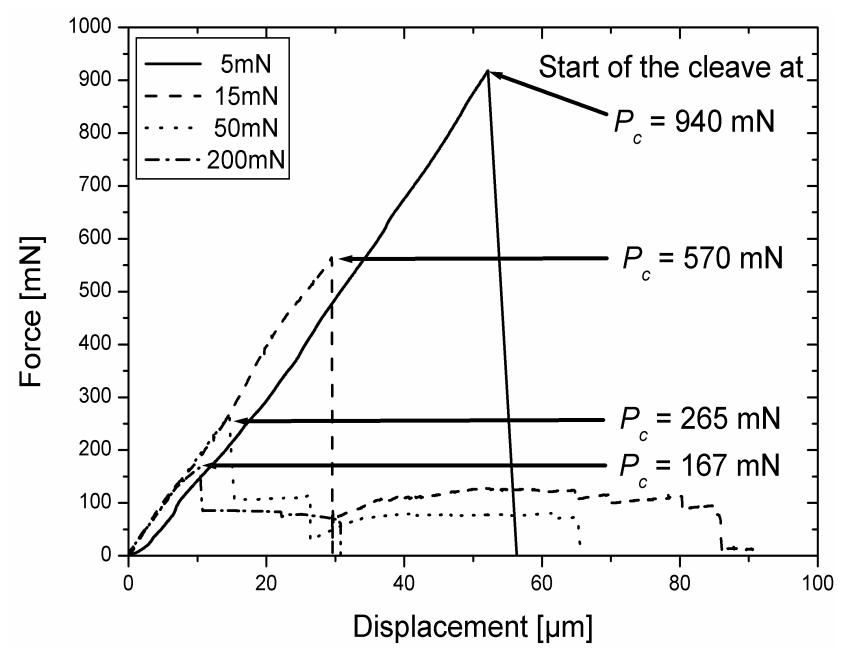

Figure 9: Results of the "displacement-controlled" measured during the in-situ cleavage operation

\section{Analysis of the Results}

To analyse the results obtain from the in-situ scratching experiment, the tip geometry and radius must be taken into consideration. The cube corner diamond tip having a tip radius of $\approx 0.5 \mu \mathrm{m}$, it is clear that the results given in Table 2 and Figs 3 and 4 are due to the faces of the diamond tip since the tip radius in these cases is small compared to the penetration depths. In contrast, the Berkovich tip having a tip radius of $\approx 2.5 \mu \mathrm{m}$ implies that until a depth of $0.1 \mu \mathrm{m}$ corresponding to a scratching force of $5 \mathrm{mN}$, the deformation process can be considered as spherical. At deepest penetration the deformation process changes and the effect of the three sides is predominant. This will also have an influence on the onset of radial and laterals crack as well as the generation of chipping out. The origin of the larges chips is the intersection of the lateral with the radial ones as shown in Figs 3 (b) and 4 (b). Based on the in-situ observations, it was mentioned that when scratching edge first the particles are only generated in front or the side of the tip whereas for face first it may take place also at the behind the tip. This is certainly due to the fact that, for edge first, the material is pushed away more smoothly compared to the others. In contrast, for face first, the material is "stuffed" away by the edges introducing higher stresses and friction. Having not a straight line when scribing with the CC tip face first in Fig. 4 (a) seems to indicate that the Nanoindenter-XP has a low lateral stiffness.

The force-displacement curves presented, in Fig. 9, show clearly a correlation between the $P_{S}$ and a, and so $P_{C}$. Actually, the values of $P_{C}$ rise as $P_{S}$ decrease and this is consistent the fracture mechanism principles. In order to determine the relationship between $P_{S}$ and $P_{C}$, a comparison between the ratios at 15 and $50 \mathrm{mN}$ is made for a in $\mu \mathrm{m}, P_{S}$ and $P_{C}$ in $\mathrm{mN}$ which gives the following results: $\frac{a_{50}}{a_{15}}=\frac{15.33}{4.49}=3.4$ for $a$ and $\frac{P_{S 50}}{P_{S 15}}=\frac{50}{15}=3.33$ for $P_{S}$. In order to be able to compare these results with the ones obtained from the critical load, it is necessary to take into account the inversely proportionality between the crack depth and the critical load. In other words, larger is the defect, lower is the force required for the crack to initiate. Consequently, the ratio $\frac{P_{C} 15}{P_{C 50}}=\frac{570}{265}=2.1$ must be employed. Another comparison possible is to make use of stress intensity factors calculations. Indeed, assuming the flaw having a semi-elliptical shape, it is possible to calculate the stress intensity factor $K$ from national standards or in-houses procedures which allow to predict crack propagation such as the French A16 [13] and British R5 [14] and BS7910 [15]. Hence, knowing the initial dimension of the median cracks and the $K_{I C}$ for GaAs $(0.31[11])$, it is possible to determine the force needed for the crack initiation. Since the loading condition are not exactly similar, a direct comparison is not possible but a good approximation can be obtained by comparing the $P_{C}$ ratio calculated previously with the one obtained between $P_{K_{I C} 50}$ and $P_{K_{I C} 15}$. Taking into account the initial size defect for both scratching conditions, the loads necessary to reach $K_{I C}=0.31$ [11] were computed from the French procedure A16 [16]. The result is given by $\frac{P_{K_{I C} 50}}{K_{I C} 15}=3.4$. This number is not in line with the one of 2.1 from the experiment but is consistent with the linearity obtained between $a$ and $P_{S}$. Two causes may be responsible for these discrepancies. The first is that Eq. (2) [12] and the French procedure [16] do not characterise perfectly the experimental loading case. Eq (2) is only for a semi-elliptical defect in the middle of plate in pure tension. The A16 procedure is derived for a plate containing in its middle a semi-elliptical flaw in 
superimposed axial and bending loads and thus closer to our test configuration. The second reason is the scatter existing in the depth of the initial median crack due to scratching. In any cases, it can be concluded that these results are in good agreement with the theoretical calculations.

It is interesting to notice that the values of $P_{C}$ for GaAs $-5 \mathrm{mN}$ in Fig. 9 is only of $940 \mathrm{mN}$. This is surprising since no initial defect was measured as evident from Fig. 7. On the other hand, it is known from the penetration curve that between 4 and 15 $\mathrm{mN}$, plastic deformation is already present. Hence, if $P_{C}$ is not higher, it means that although there is no initial crack until 15 $\mathrm{mN}$, the cleavage process and behaviour is influenced by the plastic deformation.

The standard deviation in Fig. 6 increases with the loads. This can be explained by looking at the Fig. 7 (a) where at loads less than $50 \mathrm{mN}$, the depth of initial defect is well defined and is seen to be approximately constant. On the contrary, at $150 \mathrm{mN}$, for example, the assumption that the MC is constant is not acceptable as illustrated in Fig. 7 (b). In this figure, it is obvious that there are many points were stress concentrations occur which affect directly $P_{C}$ and so the disparities in the measured values.

Furthermore, three types of behaviour results from the cleavage operation. The first is that the crack does not grow through all the length of the wafer at once and part of the crack growth happens by steps as for $200 \mathrm{mN}$ in Fig. 9. In the second case, the crack just propagates throughout the length of the wafer at once as observed for the case of $5 \mathrm{mN}$ in Fig. 9. This can be explained in terms of energy in the system. Having a larger median crack requires less energy to initiate the crack. Hence, the majority of the energy released is employed for the creation of surfaces during crack growth and part of it is dissipated otherwise such as stress waves [9-10,12]. On the contrary, for small MC, a large amount of energy is needed to initiate the propagation. The amount of stored energy is thus sufficient to propagate the crack trough the length of the wafer.

Finally, these cracking behaviours influence the feature visible on the cleaved surfaces and so three behaviours can be distinguished. The first is that in some cases, although GaAs is anisotropic with a preferred $\{110\}$ cleavage plane, the crack deviates from its path and is uncontrollable. Secondly, the crack follow the $\{110\}$ cleavage plane but the surface contains some features such as river or Wallner lines [19]. Lastly, not only the crack follows its path but an atomically flat cleaved surface is obtained. The first two behaviours may occur on two grounds. (1) There is a strong shear opening stress component at the crack tip during the advance of the crack front due to a loading condition in mode III (out-of-plane - shear). (2) The influence of the crack tip speed. Actually, it was found that there is a sharp transition between a smoothly rising velocity to an oscillating velocity during the fracture process depending on the boundary conditions [9]. This is explained by the fact that a moving crack generates stress waves that spread out from the face of the crack and dissipate energy. This results in a change in the local and global stress field driving the crack growth. Consequently, as the crack front moves through a complex field of reflected waves which may produce significant changes in the fracture path and crack speed and thereby affect the cracking direction and /or the fracture surface topography $[9,12,19]$. The transition speed was found to be approximately $35 \%$ of the Rayleigh wave velocity [9]. Consequently, to have a perfect cleavage surface, it is necessary to control the crack initiation and its propagation. To achieve this goal, it is imperative to (a) avoid loading condition in mode III and (b) find the best compromise between the largest median crack possible but without containing stress concentration due to damaged surfaces.

\section{Conclusions}

In this paper, the observation of scratching and brittle fracture of semiconductors during in-situ scanning electron microscope experiments has been presented. It has been shown that in order to cleave GaAs, an initial defect must be present. This defect is defined as a median crack and it is always generated along the preferred $\{110\}$ cleavage plane for GaAs. The scratching tip direction influences only slightly the onset of radial cracks, the subsurface crack network and the chipping out. When scratching along [110], the slip bands are not visible and radial cracks are along [100]. The radial and lateral crack does not seem to be affected by the scribing velocity. It has also been demonstrated that the depth of the MC is correlated with the scratching load according to the power law, $P_{S} \propto \chi \cdot a^{3 / 2}$, often used in indentation, although the principles are much different. By taking specific values, it was established that this relation can even be linear form such as $P_{S} \propto \chi \cdot a$.

Based on the cleavage tests, three main conclusions can be drawn. First, the relation determined between $P_{C}$ and $P_{S}$ follows the $3 / 2$ power-law whereas the calculations based on the French procedure A16 [13] gives a linear correlation. It must be notice that this difference are based on only one specific case and the disparities may be due to (a) the fact that both approximations does not symbolise exactly the experimental set-up and (b) the scatter in the depth of the median crack was not taken into account. Second, the scatter in the load required to initiate the MC is dependent on its depth. At load up to 75 $\mathrm{mN}$, it was possible to create a crack with a constant depth on a length of several millimetres. At higher load, the MC became rougher and stress concentrations exist which results in large variations in $P_{C}$. Finally, two types of cracking behaviours were 
observed depending on the amount of the energy put in the system. For large median crack, the propagation is characterised by a fast initiation over a small distance followed by jumps, whereas for small MC, the crack just growth throughout the length of the wafer at once. Depending on the type of crack propagation, it was observed that cleavage may deviate from its path, or follow the $\{110\}$ cleavage plane. In this latter case, either visible features or atomically flat cleaved surfaces are obtained. This is due to either a strong shear component at the crack tip as the crack propagates and / or due to a cracking speed $\geq 0.35$ Rayleigh wave velocity.

The conclusion of this paper is that to produce laser bar diodes with atomically flat cleaved surface, two major points are required: (1) avoid loading condition in mode III and (2) find the best compromise between the largest median crack possible without damaging the surfaces or generating stress concentrators in the median crack.

\section{Acknowledgments}

The authors would like to acknowledge the provision of material from Bookham (Switzerland) AG, the financial support from the KTI / CTI project Nanoclé ( $N^{\circ}$ 6025.2) and by the Swiss Program TopNano21 (contract $N^{\circ}$ 203.269). The authors would like to thank Mr Bürki for the pictures taken from the High Resolution SEM.

\section{References}

1. Wasmer, K., Ballif, C., Gassilloud, R., Pouvreau, C., Rabe, R., Michler, J., Breguet, J.-M.-, Solletti, J.-M., Karimi, A. and Schulz, D. "Cleavage Fracture of Brittle Semiconductors from the Nanometer to the Centimetre Scale", Advance Engineering Materials, Vol. 7, Issue 5, pp: 309-317, (June 2005).

2. Scannel, D. and Smith, D., "Scribing Compound Semiconductors: An Application Primers", Feature, Report Nº ElII-VR V2NI and Karl Suss Report, (November, 1987).

3. Ure, J. W., "Application of Scribing Optoelectronic Devices", Karl Suss Report, (May 1988).

4. Le Houérou, V., Sangleboeuf, J.-C., Dériano, S., Rouxel, T. and Duisit, G., "Surface Damage of Soda-Silica Glasses: Indentation Scratch Behaviour", Journal of Non-Crystalline Solids, Vol. 316, Issue 1, pp: 54-63, (2003).

5. Bucaille, J. L., Felder, E. and Hochstetter, G., "Mechanical Analysis of the Scratch Test on Elastic and Perfectly Plastic Materials with the Three-Dimensional Finite Element Modeling", Wear, Vol. 246, Issues 5-6, pp: 422-432, (2001)

6. Bucaille, J. L., Felder, E. and Hochstetter, G., "Experimental and Three-Dimensional Finite Element Study of Scratch Test of Polymer at Large Deformations", Journal of Tribology, Vol. 126, Issue 2, pp: 372-379, (2004).

7. Rosentreter, M. A., Wenderoth, M., Theuerkauf, N. H., Heinrich, A. J., Schneider, M. A. and Ulbrich, R. G., "Cleavage and Brittle Fracture in GaAs: Indication of Lattice Dynamic Instability on the Nanometer Scale", Europhysics Letters, Vol. 38, Issue 9, pp: 675-680, (1997).

8. Margevicius, R. W. and Gumbsch, P., "Influence of Crack Propagation Direction on $\{110\}$ Fracture Toughness of Gallium Arsenide“, Philosophical Magazine A, Vol. 78, pp: 567-581, (1998).

9. Sauthoff, K., Wenderoth, M., Heinrich, A. J., Rosentreter, M. A., Engel, K. J., Reusch, C. G. and Ulbrich, R. G., "Nonlinear Dynamic Instability in Brittle Fracture of GaAs", Physical Review B, Vol. 60, Issue 7, pp: 4789-1795, (1999).

10. Okui, T., Hasegawa, S., Fukutome, H. and Nakashima, H., "Shearing Orientation Dependence of Cleavage Step Structure on GaAs (110)", Surface Science, Vol. 448, Issues 2-3, pp: 219-224, (2000).

11. Chen, C. P., "Evaluation of GaAs Fracture Mechanics ", National Aeronautics and Space Administration (NASA) Report, JPL Publication No NPO-16604/6095, pp: 1-63, (January, 1987).

12. Lawn, B. "Fracture of brittle solids", $2^{\text {nd }}$ Ed., Cambridge University Press, (1997).

13. Commissariat à l'Energie Atomique, "A16: Guide for Defect Assessment and Leak Before Break Analysis", $4^{\text {th }}$ ed. Saclay: CEA, (2002).

14. Ainsworth, R. A. Editor "R5: Assessment Procedure for the High Temperature Response of Structures", Nuclear Electric procedure R5, Issue 2, (1999).

15. British Standard, "BS 7910: Guide on Methods for Assessing the Acceptability of Flaws in Metallic Structures", BSI, (1999).

16. Rabe, R., Breguet, J.-M., Schwaller, P., Stauss, S., Patscheider, J. and Michler, J., "Observation of Fracture and Plastic Deformation during Indentation and Scratching inside the Scanning Electron Microscope", Thin Solid Films, Vol. 469-470, pp: 206-213, (2004).

17. Hjort, K., Söderkvist, J., and Schweitz, J.-A., "Gallium-Arsenide as a Mechanical Material", Journal of Micromechanics and Microengineering, Vol. 4, Issue 1, pp: 1-13, 1994.

18. Solletti, J.-M., Parlinska-Wojtan, M., Tharian, J., Wasmer, K., Michler, J., Ballif, C., Schulz, D. and Karimi, A., "Fracture Mechanisms of GaAs under Nanoscratching", in Proceeding of Materials Research Society Symposium R: Fundamentals of Nanoindentation and Nanotribology III, edited by Kathryn J. Wahl, Norbert Huber, Adrian B. Mann, David F. Bahr, and Y.-T. Cheng, Warrendale, PA, Vol. 841, pp: R9.15, (2005).

19. Hull, D., "Fractography - Observing, Measuring and Interpreting Fracture Surface Topography", Cambridge University Press, $1^{\text {st }}$ Ed., (1999). 